\title{
THE PROBLEMS EXPERIENCED By Black STROKe PatientS IN SOWETO, SOUTH AFricA
}

\begin{abstract}
A purposeful sample of Black Sowetan residents who had sustained a stroke were studied to identify the problems they were encountering after discharge back to their homes. A descriptive qualitative approach was used comprising semi-structured interviews in the subjects' homes. Audio recorded data was transcribed in extenso, and coded into themes. The data revealed that the subjects' lacked knowledge of their disease processes. However, medication non-com-

HALE LA', EALES CJ', STEWART A', FRITZ VU ${ }^{2}$

\author{
1 Deportment of Physiotherapy, University of the Witwatersrand \\ Johannesburg \\ Johannesburg
}

2 Department of Neurology, University of the Witwatersrand, pliance was largely due to financial and transportation difficulties in attending clinics. Although most of the sample was able to walk, they felt the need for improved walking ability, as they were scared of falling. Most were independent in $A D L$, yet they, and their families perceived them to be otherwise, leaving the subjects with a sense of worthlessness. Pain in the shoulder and stiffness were the common secondary problems encountered. There is a need to educate stroke victims with regards to their disease, it's secondary complications and their capabilities following stroke. Safe walking must be ensured before discharge.
\end{abstract}

\section{KEY WORDS: STROKE, PROBLEMS, EDUCATION}

\section{INTRODUCTION}

"In rehabilitation we seem to be relatively successful in understanding what life experiences mean to our patients, so long as they are people like ourselves who share the middle-class American belief that one should value independence. We may have great difficulty, however, when working with persons who do not share this cultural belief and whose life experiences have been based on an interdependent pattern of working co-operatively with others in performing many life activities... It is the individual's experience of disability as interpreted within his or her personal world, not medical status or biology, that gives meaning to the actions of rehabilitation" (Spencer, 1993).

Present stroke rehabilitation programmes in South Africa may be inappropriate (Hale and Wallner, 1996). To redress the situation, a survey is being conducted in Soweto, South Africa to

\section{CORRESPONDENCE:}

\section{Mrs LA Hale}

Physiotherapy Department, Faculty of Health Sciences University of the Witwatersrand 7 York Road, Parktown, 2193, South Africa ascertain the problems facing stroke victims post-discharge. A specifically designed questionnaire is being used in a structured interview process (Hale et al, 1998a). This questionnaire is based on the training and experiences of one of the authors, and thus may reflect this author's perceptions of the problems, and not those of the subjects. This emic approach which is the author's perception of reality, may in fact not be the reality of the situation, this would then be the etic approach. The author may be biased by the training received via curricula developed out of the environmental context of the subjects being investigated. The designed questionnaire, although useful from a quantitative aspect, may not be identifying issues that the subjects themselves may feel are important.

Phenomenology was a philosophical approach to research that developed in the mid-1800s in Germany. This approach attempted to understand social phenomena or human activity from the viewpoint of the person being studied. The term was later refined to that of hermeneutics, Hermes being a Greek god who acted as an interpreter (Shepard et al, 1993). Inclusion of a hermeneutic perspective to a survey of this nature was considered essential.
The phenomenologist believes that individuals need to be understood in their entirety within a situational context, not separated from the environment in which they function (Shepard et al, 1993). Thus the qualitative aspect of this survey should be in the form of in-depth interviews conducted in the subject's homes by the researchers themselves.

\section{RESEARCH QUESTION}

To conceptualise and describe the problems encountered by Black Sowetan residents who had sustained strokes and were now back living in their homes.

\section{CONCEPTUAL FRAMEWORK}

Figure 1 conceptualises the authors' perception of the problems facing Sowetan stroke victims.

\section{SAMPLE}

In qualitative research, the method of purposeful sampling is employed. Subjects are strategically chosen for a specific purpose, rather than randomly as in the case of quantitative research. Fewer subjects are used than in qualitative research, as data is accumulated in depth rather than in quantity. It is difficult to predict how many subjects are required to be sampled, as it depends on the thickness of knowledge gained from 


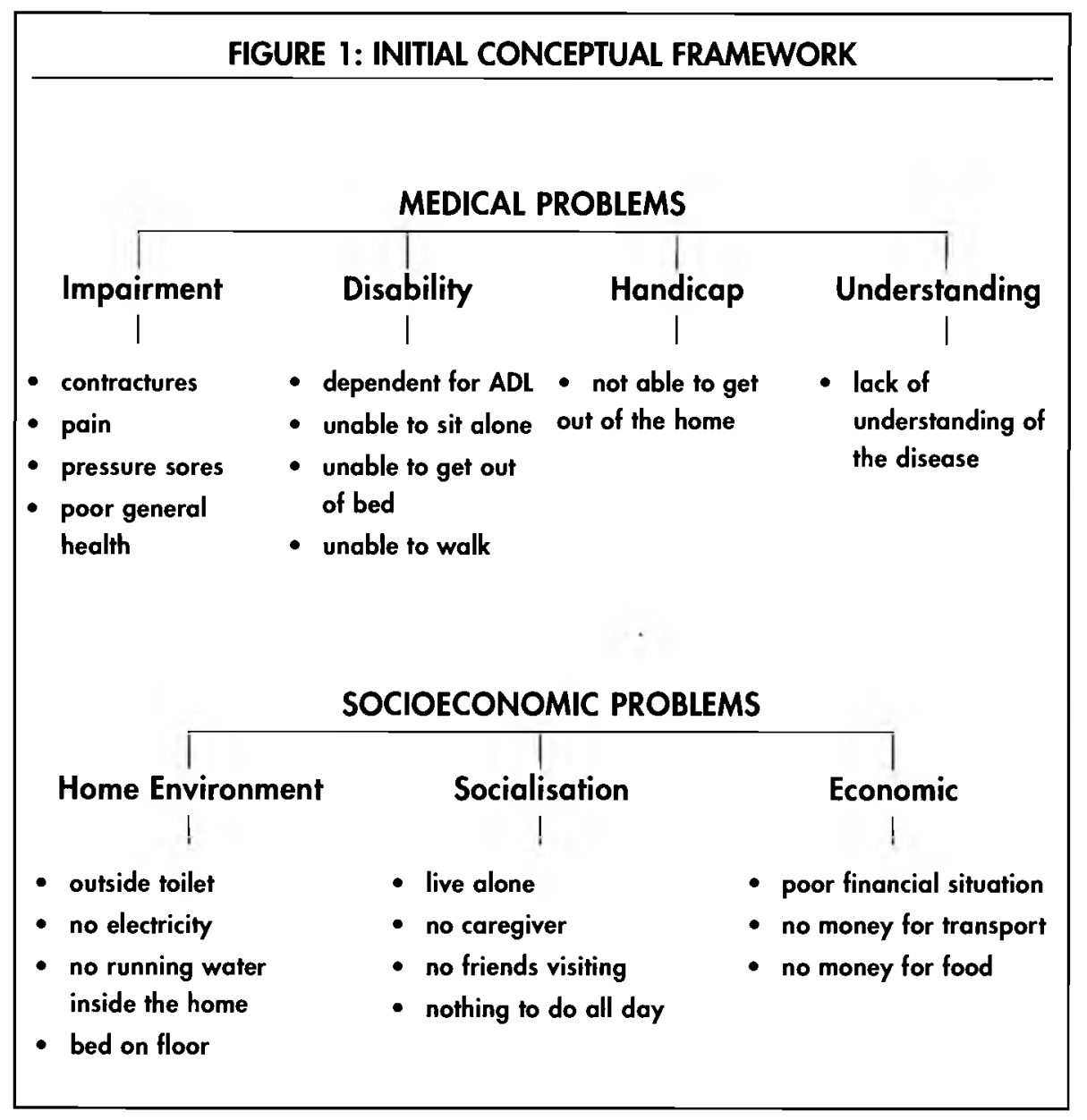

each individual subject. Sampling is completed when researchers feel that they have an in-depth knowledge of the phenomenon being studied.

Stroke victims had been identified from the medical admissions ward of the $\mathrm{CH}$ Baragwanath Hospital for inclusion into the quantitative survey mentioned above. The identified subjects had been interviewed in their homes at three months post-discharge. Subjects having the following problems were identified:

- Those who were unable to walk out side of their homes

- Those who were unable to use their affected arm functionally

- Those who indicated from the initial interview that they had one or more of the following impairments: pain, pressure sores, or contractures

Subjects were purposefully sampled in order to gain a better understanding of their problems.

Additional criteria were that the subject should own a telephone and live in formal housing. The reasoning for this was logistical, so that appointments could be made, and addresses easily found.
Two final year physiotherapy students were included in the first two interviews in order to obtain their independent observations as additional triangulation of data. The students were instructed to write up their observations of the visits independently. One student was asked to concentrate on observing the home environment, while the other student focussed on the subject's non-verbal communications, which is not registered by an audiotape recording (In order to prevent bias, the interviewing author only read the student observations after she had studied the taped interviews.)

On arrival at the subject's home, the customary introductions and greetings were exchanged. Verbal informed consent for the visit was confirmed, as well as permission to an audiotape recording of the interview. This permission was repeated for the records once the tape was running.

The interview was conducted and taped by the interviewing author inside the home of the subject, most frequently in their living rooms, with all concerned seated comfortably. The interpreter and the students only interjected if a translation was required. They were given an opportunity at the end of the interview to ask any questions of their own. Although this was an unstructured interview, the subject being allowed to speak at will, a few questions were pre-set in order to initiate the conversation, for example: "What would you really like to be able to do?"; "What would you like help with most?"; and "What worries you most about your stroke?"

The audiotapes were transcribed in extenso by an independent party.

The data was analysed using phenomenographic methodology (Merriman, 1988; Strauss and Corbin, 1990). Line by line analysis of the transcripts was done to establish common concepts. These concepts were then grouped into categories, and coded in such a way as to reduce the data (open coding). Axial coding was then undertaken to make connections between the categories in the open coding so that the most important themes in the data could be identified.

\section{RESULTS}

Using the selection criteria described above, thirteen subjects were identified for the qualitative interviews. However, 
TABLE 1: DISABILITY PROFILE OF THE SUBJECTS

\begin{tabular}{|l|l|l|l|}
\hline & $\begin{array}{l}\text { Barthel Index Score } \\
\text { |scored out of 20| }\end{array}$ & Severity at discharge ${ }^{2}$ & $\begin{array}{l}\text { Ambulant at time of } \\
\text { qualitative interview }\end{array}$ \\
\hline RM & 4 & dense & no \\
\hline JN & 12 & mild & yes \\
\hline MK & 17 & mild & yes \\
\hline SM & 11 & mild & yes \\
\hline AM & 11 & dense & no \\
\hline Lim & 9 & mild & yes \\
\hline GK & 15 & moderate & yes \\
\hline EM & 14 & moderate & yes \\
\hline ER & 19 & moderate & yes \\
\hline
\end{tabular}

it was subsequently found that four of them had passed away. Having conducted nine qualitative interviews it was felt that sufficient data had been obtained. The age of the subjects ranged from $44-76$ years, with a mean age of 64 years. The group comprised two males and seven females, of whom five subjects had sustained a right cerebrovascular accident and four, a left. The disability profile of the subjects can be seen in Table 1 . In all subjects, English was not their home language.

\section{DETERMINING TRUSTWOR'THINESS OF}

\section{THE DATA}

Trustworthiness or the reliability of data was determined by the following methods:

1. Use of thick descriptions built on low inference data, the exact language of the interviews was analysed, for example: "Stroke is a funny thing. you can't know anything, I don't know where that it comes from."

2. Triangulation of the data was obtained by looking for similarities found in multiple data sources: that obtained from the qualitative interviews; the structured interviews performed in the quantitative survey at three months post-discharge (Hale et al, 1998a); data from the hospital medical records; and the recorded observations of accompanying physiotherapy students. Consistency was found in the majority of cases.

4. Multiple subjects were interviewed.

5. Reliability check of data coding. Interreliability tests were carried out on all nine interviews by a second researcher, trained in qualitative research. An average agreement of $87 \%$ with the original categorisation was found. A few categories were changed after some discussion by the coders. This ensured that the codes were understandable, exhaustive, mutually exclusive and independent.

Unfortunately it was not possible to return to Soweto to enable the subjects to check (member check) the transcriptions of their interviews.

\section{THEMES}

\section{Lack of knowledge regarding their condition}

A recurring theme with most of the subjects interviewed was their lack of knowledge regarding their condition and its treatment. They all knew that they had had a stroke, however five of the the stroke was.

The conversation went much like this:

Question: "Do you know why you had the stroke?"

Answer: "No."

Question: "Do you know what caused it?"

Answer: "No I don't know."

Question: "Did the doctor's tell you why?"

Answer: "The high blood."

Question: "Do you think that caused your stroke?"

Answer: "I don't think so."

And from another subject:

Question: "And do you think that the high blood pressure has any thing to do with your stroke?"

Answer: "I don't think so, I don't know, it just happened." subjects were not sure what the cause of
One subject was sure that his stroke was due to hypertension and the fact that he had run out of medication which he simply could not afford. He lived in the best home of all, read a lot and listened all day to talk shows on the radio, implying perhaps that his general education was better, and thus, perceivably his understanding of his condition. One subject thought that his stroke might have been due to hypertension, but he felt it was more likely due to his heavy smoking. Again, this subject appeared more educated than the others. He also said that he had given up smoking: "I can't force the thing that wants to kill me."

Six of the subjects felt that their stroke was due to stress and worry (patients' own words)

"When I had the stroke maybe I was worried because of my elder son. He was in jail, I was worried about him"

_ "The doctor's say to me that I am thinking too much. Yes, I was worried about my mother."

- "I think it was because I was too worried about my daughter"

- "I had one because I was thinking too much - I had a lot of worries at the time."

- "I was thinking a lot, a lot of problems. I had problems about my late daughter."

One subject was not even aware she was hypertensive. Her granddaughter knew and monitored her medication. She had no idea of the cause of her stroke: "Really I don't know what is a stroke. I don't know what has happened. Me, I said maybe because every day I have got what I call the feeling. Maybe it is that feeling there."

All nine subjects were hypertensive. They were all on medication, and vaguely knew what it was for. They knew that they had to take their medication. Noncompliance was usually due to the inability to get to the clinics and a lack of finances. One lady lived around the corner from the clinic, but being unable to walk had to order a taxi to fetch her from the house. The taxi then had to wait at the clinic till she was finished in order to bring her back. This cost her R20.00, a substantial amount for an old age pensioner (she had not being getting her pension for a few months). 


\section{Gait}

Seven of the nine subjects could walk, most of them with the assistance of a stick. Two subjects were bedridden. A common thread to this theme was a great fear of falling, especially while alone in the home. They felt strongly that: "I shouldn't be clone in the house".

Walking to an outside toilet appeared to be a good test of a person's gait capability. All had to negotiate one or two steps from their kitchen doors, then walk a few metres along a path of impacted (hard) soil to the toilet (all were of the raised, flushing variety). Although six out of nine subjects with outside toilets were able to walk with a stick, two felt that they had to have stand-by assistance in order to walk to the toilet. All the others were independent. In spite of this functional walking ability, six of the subjects expressed the desire to be able to walk better:

"I would like them to help me with my walking. I would like to walk like normal people, I can walk but I am not happy with the way I walk", and yet another: "I want to walk, I want to move, I can't sit still the whole time".

"I am worrying, but maybe if I can have a stick I can walk better - I need a big stick".

One of the bed-bound subject's greatest wish was to walk: "That I should be able to walk at least. I must be able to walk."

\section{Activities of daily living (ADL)}

With the exception of the bed-bound subjects, most of the subjects were independent in ADL. Three needed help with washing (especially with washing the non-affected arm). One had been unable to wash herself for awhile, and was extremely relieved that she could now manage: "It is a shame for my children to wash my body." Dressing is another ADL task that required help (three subjects): one subject complained of being unable to lift up her arms, and one lady said that she stays in her nightgown all the time, as it is the easiest piece of clothing to put on.

Although most subjects were independent in ADL, they were concerned about their perceived loss of independence: "I can't do nothing. I can't do anything because I can't walk, I can't just move myself. I have got to be helped all the time." And yet another subject: "I said no I am not here to say 'please'. In the night that worries me." In other words, she was determined to be independent, but worried that she could not actually manage.

\section{Upper limb function}

Interestingly enough, five of the subjects had voluntary movement of the hand, which they appeared to try to use. A few expressed the desire to be able to use their hands:

"It is only the hand, it can just be that I can even pick it up or to move it." and "the hands they must hold." However, the desire for improved gait function was mentioned far more than that of regaining upper limb function

\section{SECONDARY COMPLICATIONS OF THE STROKE:}

The main secondary complications were pain, swelling of the feet, and stiffness. Three of the subjects complained of stiffness in the upper limb, and had the potential to develope contractures: "I feel stiff, stiff, if I want to stretch myself I can't stretch, and I just sit". Only one subject suffered from pressure sores, the old bed-bound lady with diabetes. The daughters were struggling to heal them, their mother being very immobile and obese. They did, however, have an understanding regarding pressure sore development.

Shoulder pain, and sometimes hand and elbow pain was a frequent complaint. Seven subjects complained of having shoulder pain. Two subjects complained of pain in the knee, especially on walking. They also complained of their hips feeling "loose", the exact meaning of this was not elicited: "The main thing is my hip. I think it is loose because when I step it is shaking like this".

\section{THE PSYCHOSOCIAL OUTCOMES}

\section{Housework}

Most of the subjects were not able to help much in the home, and this was of great concern to some of them: "I always fight with my family, let me do something, not just sit". Some helped with washing the dishes whilst sitting down; others could make their beds and make simple meals or a cup of tea in the kitchen. They washed their own under- wear, although hanging it up was often a problem. There was a great desire to be useful: "Now I am sad for myself because when I could do things for myself, I could work for my children, I could make everything for my children but now I can't." The subjects expressed this need many times: "Maybe they can help me to help myself and wash myself, so that I can try to cook for myself", and "The washing, I would like to do the washing. Washing myself and the clothes."

\section{Socialisation}

Most of the subjects were not occupied during the day. Few people came to visit, and only two subjects actually went to visit their neighbours. Most of the subjects listened to the radio or watched TV. The gentlemen who was bed-bound, sits on his own all day (locked into the house for security reasons) and listens to talk shows on the radio. The bedridden old lady disliked radio and TV, and cried often out of loneliness. A]though she had family around, it appears that she wanted someone of her own age to talk to. The daughters are now considering placing her in an old age home. Only one subject was able to work again - he had his own business, but it involved lifting objects, which he found difficult. However, none of the subjects were actually living alone.

\section{Financial concerns}

A recurring theme was the financial problems that all the subjects had. Many were pensioners, and the interviews took place at a time when the South African government was implementing a policy of checking the legitimacy of all pension claims. This resulted in a backlog of payments, adding to the already strained resources of the subjects. Some of the subjects had been the sole source of family income prior to their stroke and now found themselves and their families in dire straits. Only one of the subjects had had a medical aid scheme but it had subsequently dried up.

The lack of finances resulted in transport difficulties, as most of them had to catch mini-bus taxis. As they were often unable to walk to a taxi rank, the taxi had to fetch them at home, adding to the cost. In addition, there has been the complaint from a few sources that taxi drivers charge more for disabled people 
as they are slow to get on and off, and their walking aids or wheelchairs take up too much room.

Two subjects were anxious to return to work: "About work. The others go to work and I stay by the home, that worries me." and "What worries me about my stroke is that I haven't got so much power to work because I am still not quite right"

\section{Motivation}

Many of the subjects were religious, and put a lot of faith in the powers of prayer: Question: "so how did you get so much better?" Answer: "I prayed my Lord. I did pray hard."

Some showed great determination to get better:

"I am struggling to do it, I am trying, I force it. I always fight with my family, let me do something, not just sit."

And ... "Myself, I was fighting very hard.... Yes, you know because once you have got the stroke you don't want to do something painful, punish yourself, and when you have got stroke moving is painful but if you leave it, it just stays, you have to force that pain to go, you must force it like this, you must force it yourself, the muscles are getting weak because the muscles are stiff, if you leave like that then you will have to go with the wheelchair, but if you want to live, then you must force it."

Unfortunately, the discussion did not go deeply enough to extract any cultural beliefs on the subject of stroke.

\section{DISCUSSION}

With the information emerging from this study, the conceptual framework, drawn up prior to the study, was then revised, and is displayed in Figure 2.

From the revised conceptual framework, it becomes evident that many of the items the author felt may have a negative impact on the patient's life, actually had a positive impact. For example, the patients were not alone, they lived with family; the extended family often providing tremendous support. This may negate the lack of socialisation with friends. The high rate of unemployment in this area probably means that there are more adults around to support and give company during the day. The concept of an extended family may work in stroke victims' favour, as Spencer wrote (1993):
"It is our expectation that persons with an interdependent way of life prior to onset of disability may adapt more readily because they are accustomed to giving and receiving assistance and doing things cooperatively and thus expect this as a natural way in which things are done in their local world."

Although the interviewing author felt empathy for the subject's apparent lack of socialisation, this was not expressed by the subjects themselves. The bed-bound gentleman who spent most of the day, and possibly the night, alone, expressed a desire to walk again and not to socialise more. The extended family of these subjects may, perhaps, play a far more positive role in socialisation of the subject than does the isolated family units in Western cultures.

Rather than becoming demotivated, the patients appeared to have a high level of motivation, with religion playing an important role in this regard.

It seems that the factor most worrying the subjects after a stroke is not the inability to walk, but the need to be able to walk safely without fear of falling. The early recovery of walking has been noted in the literature (Wade et al, 1987; Jorgensen et al, 1995; Hale and Eales, 1998b), however, these studies have been quantitative, and thus the fear of falling has not been highlighted. This fear persists in spite of most of the subjects having sticks with which to walk. In this sample group, knee pain after walking was not common, but some of the subjects felt it was a problem. Having observed many of them walking with abnormal gait patterns, it is questionable whether pain free ambulation will persist. However, the subjects did not actually have to walk far, their properties being small, and none of them expressed the desire to walk further out of the property, say to visit friends. It is difficult to decide whether the fear of falling prevents further excursions, or the lack of desire does so.

Interestingly enough, many subjects had return of upper limb function, and only some expressed the wish for it be improved. However, there was a high incidence of pain in the shoulder. The other secondary complications of stroke did not seem significant, except for the stiffness which most likely would develop into contractures at a later stage.

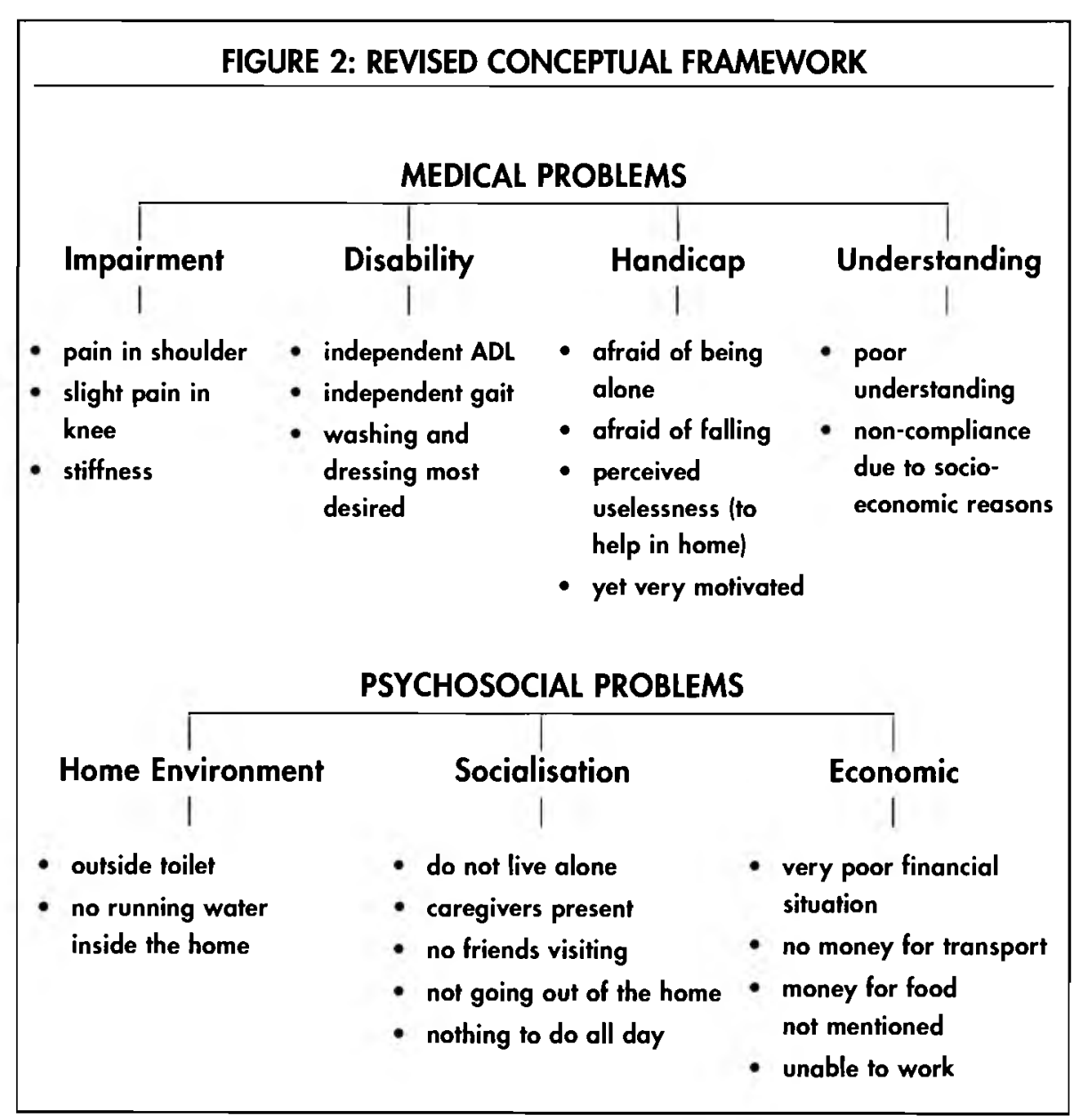


Most of the patients were mobile and independent in ADL. They did get out of bed and get to the toilet. The functional task most desired appears to be the task of washing oneself. Only three houses had proper bathrooms with running water. Most of the subjects would have to fetch water from the kitchen tap, heat it up and use it to wash in a tub. However, it was not so much the lack of independence in activities of daily living but the loss of usefulness, of worth, within the family unit which the subjects found so distressing. They would like to have some meaningful role, to be allowed this role, in the home, for example, to do some cooking or wash the clothes.

Education would appear to be the main form of intervention required. Although the subjects and their families were mostly aware of what a stroke is, there appeared to be a lack of insight into what causes stroke. There was little understanding of the risk factors. The subjects were aware, in general, of their medication. Non-compliance appeared to be due to a lack of financial resources to get to clinics. These facts have been highlighted in a previous studies (Hale et al, April 1998; Stewart et al, 1999). Some of the subject's complained of short-term memory loss, which would have to be taken into account when planning education strategies.

\section{CONCLUSION}

Although there was a possible lack of depth to the interviews, and much information may not have been elicited, the study was still revealing. It highlighted many areas of concern that would need to be addressed in a rehabilitation program. Education is extremely important. The stroke patient, and for that matter, the general public, need to be enlightened about stroke: what it is, it's risk factors and it's prevention. In addition, the patients and their families need to be taught on how to prevent the secondary complications of stroke, especially with regards to pain and contractures, as well as being taught on what they can and should not do. For example, the family must encourage the patient to be able to fulfil a role in the home, and not be discouraged from working in the home. Relearning of independence in ADL, especially in dressing and washing would help tremendously to improve the patient's self-esteem. Assisting a patient to walk safely is a priority.

Unfortunately, the financial burdens of the patient and their families can not really be addressed by the physiotherapist, apart from appropriate referral. Rehabilitation has to take place in the context of the patient's life - his home and his financial state - wishing for more is unrealistic. However, there is a very valuable attribute that be can harnessed in the rehabilitation of people like those interviewed in this study, and that is their motivation, and in many cases, their deep religious faith.

One subject felt very strongly about the need never to give up: "They must fight with some exercise, and they must never, never relax. They must not think that when they go to the hospital for the stroke that they are going to give a special medicine for the stroke, very much the special medicine for the stroke is exercise, exercise, that's all".

Another subject, an old lady, was moved to say, in spite of her own disabilities: "You know when I got there (Zola Clinic), I said, please God help me. I was crying very hard, the other people are so worse than myself, they can't even wave their hands. I feel so sorry for them."

\section{ACKNOWLEDGEMENTS}

The authors would like to thank the subjects, the two participating physiotherapy students and the interpreter for their help in making the study possible, and to Professor K Shepard, Temple University, Philadelphia, for all her guidance.

This study was granted ethical clearance from the Committee for Research on Human Subjects, University of the Witwatersrand: No. 950 111. The University of the Witwatersrand and the Medical Research Council of South Africa funded the study.

\section{REFERENCES}

Collin C, Wade DT, Davies S, Horne V 1988 The Barthel Index: a reliability study. International Disability Studies 10 (2): 61 - 63.

Hale LA, Eales CJ 1998 Recovery of walking function in stroke patients after minimal rehabilitation. Physiotherapy Research International 3 (3): 194 - 205.

Hale LA, Eales CJ, Fritz VU 1998a The Soweto Stroke Questionnaire. South African Journal of Physiotherapy 54 (4): 16 - 20.

Hale LA, Fritz VU, Eales CJ 1998b Do stroke patients realise that a consequence of hypertension is stroke? South African Medical Journal April 88 (4): 451 - 454.

Hale LA, Wallner PJ 1996 The challenge of service provision in South Africa for patients with hemiplegia. Physiotherapy 82 (3): 156 158 .

Jorgensen HS, Nakayama H, Raaschou HO, Olsen TS 1995 Recovery of walking function in stroke patients : the Copenhagen Stroke Study. Archives of Phsyical Medicine and Rehabilitation 76: 27 - 32

Merriman SB 1988 Case study research in education - a qualitative approach. Jossey Bass Pub San Francisco, London.

Shepard KF, Jensen GM, Schmoll BJ, Hack LM, Gwyer J 1993 Alternative approaches to research in physical therapy: positivism and phenomenology. Physical Therapy 73 (2): 88 101.

Spencer JC. 1993 The usefulness of qualitative methods in rehabilitation issues of meaning, of context, and of change. Archives of Physical Medicine and Rehabilitation 74:119 $-126$.

Strauss A, Corbin J 1990. Basics of qualitative research. Sage Pub Inc Newbury Park Cal.

Stewart AV, Eales CJ, Shepard K 1999 The health behaviour of black hypertensive patients at a chronic diseases clinic in a depressed socio-economic area of Johannesburg, South Africa. South African Journal of Physiotherapy 55 (1): $11-17$.

Wade DT, Wood VA, Heller A, Maggs J, Hewer RL 1987 Walking after stroke. Scandinavian Journal of Rehabiliatation Medicen 19: 25 - 30. 